\title{
Bendamustine plus rituximab: is it a BRIGHT idea?
}

\author{
Carlo Visco ${ }^{1}$, Francesca Maria Quaglia ${ }^{1}$, Chiara Bovo $^{2}$, Maria Chiara Tisi ${ }^{3}$, Mauro Krampera ${ }^{1}$ \\ ${ }^{1}$ Department of Medicine, Section of Hematology, University of Verona, Verona, Italy; ${ }^{2}$ Medical Direction, University Hospital of Verona, Verona, \\ Italy; ${ }^{3}$ Cell Therapy and Hematology, San Bortolo Hospital, Vicenza, Italy \\ Correspondence to: Carlo Visco, MD. Associate Professor of Hematology, Department of Medicine, Section of Hematology, University of Verona, P.le \\ L.A. Scuro 10, 37134 Verona, Italy. Email: carlo.visco@univr.it. \\ Provenance and Peer Review: This article was commissioned and reviewed by the Section Editor Xinyi Du (Department of Hematology, Northern \\ Jiangsu People's Hospital, Yangzhou, China). \\ Comment on: Flinn IW, van der Jagt R, Kahl B, et al. First-Line Treatment of Patients With Indolent Non-Hodgkin Lymphoma or Mantle-Cell \\ Lymphoma With Bendamustine Plus Rituximab Versus R-CHOP or R-CVP: Results of the BRIGHT 5-Year Follow-Up Study. J Clin Oncol \\ 2019;37:984-91.
}

Submitted Sep 20, 2019. Accepted for publication Oct 14, 2019.

doi: $10.21037 /$ cco.2019.10.03

View this article at: http://dx.doi.org/10.21037/cco.2019.10.03

Flinn et al. have reported the results of the long-term follow-up for the BRIGHT trial, an international study which compared the efficacy and the safety of bendamustine plus rituximab (BR) with either rituximab plus cyclophosphamide, doxorubicin, vincristine, and prednisone (R-CHOP) or rituximab plus cyclophosphamide, vincristine, and prednisone (R-CVP) for treatment-naive patients with indolent non-Hodgkin lymphoma (iNHL) or mantle-cell lymphoma (MCL). A first report describing the primary objective of the study has been previously published (1), with $\mathrm{BR}$ that was non-inferior to R-CHOP/R-CVP in terms of complete response $(\mathrm{CR})$ rate $(\mathrm{P}=0.0225$ for noninferiority). Non-inferiority was not reached when authors compared treatment arms in iNHL patients, it approached significance in the follicular-lymphoma subset, while superiority was demonstrated when authors compared BR with standard therapy in MCL patients (CR-rate ratio: 1.95; 95\% CI: 1.01-3.77; $\mathrm{P}=0.018 ; 22$ patients received R-CHOP, 11 patients received R-CVP). Overall, the safety profile of $\mathrm{BR}$ was reported to be distinct from that of R-CHOP/ R-CVP.

When the results of the BRIGHT study were published in 2014, the sole evidence of BR superiority as compared with R-CHOP and R-CVP was achieved from the study group of indolent lymphomas (StiL) non-Hodgkin lymphoma (NHL) 1 study (2). Therefore, the results of the long-term outcomes for those patients treated with $B R$ versus patients treated with $\mathrm{R}-\mathrm{CHOP}$ and $\mathrm{R}-\mathrm{CVP}$ in the BRIGHT study were eagerly awaited. As the Stil1 trial, the BRIGHT study (3) included patients with follicular lymphoma (grade 1 or 2, excluding grade $3 \mathrm{~A}$ ), lymphoplasmacytic lymphoma, splenic marginal zone B-cell lymphoma, extranodal marginal zone lymphoma (MZL) of mucosa-associated lymphoid tissue type, nodal marginal zone B-cell lymphoma (371 patients with iNHL), or MCL (74 patients). Forty-three percent of BR patients and $45 \%$ of R-CHOP/R-CVP patients received maintenance therapy with rituximab, although not on a randomized basis but following institutional practice. The results in terms of progression-free survival (PFS) and duration of response (DOR) consistently favored the BR regimen compared with R-CHOP/R-CVP, with the subset of MCL patients achieving the strongest benefit from the combination. However, it is fair to say that, although these results represent a decent picture of real life patients, the study was not powered for comparisons between the different groups of treatment in the time-to-event end points. Indeed, follow-up data were collected every 12 months with a reduction, as for an amendment of the protocol three and a half years later, to 6 months intervals. No interval for imaging during the follow up was prescribed by the protocol, and investigators could follow their established institutional practice, as no independent review committee was present.

Taken all histological subtypes, the StiL-1 trial had reported a median PFS for BR of 69.5 months, as compared 
with 31.2 months for R-CHOP/CVP. In the BRIGHT study the corresponding PFS values appeared much longer, especially for the R-CHOP/R-CVP group (5 years-PFS of $55.8 \%$ ), with median PFS rates after random assignment that had not been reached (5 years-PFS of $65.5 \%$ for patients treated with BR). Although it is always unfair to compare different studies and study populations, baseline characteristics between the StiL-1 and the BRIGHT patients did not seem to diverge so much to justify such a PFS difference. BRIGHT patients were slightly younger (median age 59-year-old versus 63 for the StiL-1), but the distribution of clinical features at presentation (Ann Arbor stage, Follicular Lymphoma International Prognostic Index, B-symptoms) as well as the percentage of patients with MCL, were pretty similar between the two studies. Such difference in PFS might be due to two main reasons: (I) the less frequent visits and radiological checks that patients in the BRIGHT trial underwent, causing disease relapse or progression detection at the time of symptoms onset, and rarely by radiological imaging; (II) in the BRIGHT study, in both treatment groups, as specified above, a significant percentage of patients received maintenance rituximab, which prolonged PFS. It is hard to evaluate the actual contribution of maintenance therapy to the observed enhanced outcome, because the type of maintenance regimen was not chosen on a randomized basis but according to the judgment of the researcher.

PRIMA (4) and MAINTAIN trials have established that the addition of rituximab maintenance significantly improved results after chemoimmunotherapy in follicular lymphoma, and further ameliorated the outcomes achieved with the $\mathrm{BR}$ regimen (5).

The phase III GALLIUM trial (ClinicalTrials.gov identifier: NCT01332968) (6) is among the most extensive studies ever performed in follicular lymphoma, including 1,200 patients. The study randomly assigned patients treated with standard induction chemotherapy to either rituximab or obinutuzumab, a third generation glycoengineered type 2 anti-CD20 monoclonal antibody. The median followup was of 3 years. PFS for obinutuzumab-chemotherapy was statistically better than rituximab-chemotherapy $(80 \%$ versus $73 \%$, respectively) while overall survival was similar between the two arms. Surprisingly, in the GALLIUM trial an accurate evaluation of safety reported a substantially higher fraction of fatal adverse events in those patients treated with bendamustine as compared with $\mathrm{CHOP}$ or CVP. The majority of toxic deaths that were observed in the bendamustine-treated group occurred during maintenance, with proportionally similar values between patients treated with obinutuzumab and/or with rituximab. The excess of mortality was reported among patients with comorbidities, with an age over than 80 years, or with a poor performance status. We certainly need to consider that we have a lot of experience with R-CHOP, but much less long-term experience with bendamustine. Bendamustine is known to be well tolerated in the short term, but its longterm toxicity profile might not yet be fully described and/ or understood, particularly in regard to cardiac toxicity, secondary neoplasms, prolonged immunosuppression.

As for the MCL setting, BR has been confirmed by the BRIGHT study long-term results to be superior to $\mathrm{R}-\mathrm{CHOP}$ as induction therapy in patients who are unsuitable for autologous transplantation. The addition of rituximab maintenance after R-CHOP (7), has significantly improved results obtained with the regimen alone, and somewhat balanced the differences in PFS between BR and R-CHOP. The attempts to build-on the BR platform in MCL have failed to demonstrate a benefit when using rituximab maintenance (8), or lenalidomide (9), with apparent increase in toxicities, including inacceptable rates of secondary malignancies. The addition of cytarabine instead, in the rituximab, bendamustine, cytarabine (R-BAC) regimen, has demonstrated remarkable efficacy but considerable hematological toxicity as compared to BR alone (10). Hematological toxicity was frequent but manageable with supportive care and judicious dose reduction. A high proportion of patients achieved a complete response, which was fast and durable, including long-lasting responders, with median DOR exceeding 3 years for most patients. The efficacy of R-BAC compared favourably with other regimens commonly used for elderly patients with MCL, with or without rituximab maintenance.

Currently, new targeted drugs are increasingly used in combination regimens, frequently followed by prolonged (and expensive) maintenance. We are eagerly waiting for results of the SHINE trial, which will tell us whether these patients can benefit of the addition of ibrutinib front-line (ClinicalTrials.gov Identifier: NCT01776840, enrollment began in Q1 of 2013).

In remaining indolent histologies (lymphoplasmacytic lymphoma, splenic marginal zone B-cell lymphoma, extranodal MZL of mucosa-associated lymphoid tissue type, nodal marginal zone B-cell lymphoma), where the use of anthracyclines has been quite abandoned by most as induction treatment, with rare exceptions (possibly nodal subtypes), BR represents the mainstay, and the results of the BRIGHT study 
support its use. Recent publications have shown how feasible and reliable is the use of 4 cycles instead of 6 in patients with MZL subtypes $(11,12)$ that achieved good clinical response. For lymphoplasmacytic lymphoma, BR also represents the most widely adopted induction treatment, although the unprecedented response rates of this lymphoma subtype to BTK-inhibition, have candidated this approach as the future standard for these patients. The presence of MYD 88 mutation is becoming important for the diagnosis of these forms, as well as in predicting tumor response, as it will probably be the case of MYD 88 mutated MZL.

New targeted drugs are becoming available for the treatment of patients with iNHL and MCL, and available data indicate that encouraging responses rates can be achieved when administering these drugs alone or in combination with chemotherapy.

The BRIGHT trial's authors must be congratulated for the effort in gathering together different histologies of previously untreated lymphoma. Taken together, Flinn and colleagues' data support the use of BR as preferred firstline treatment for iNHL and MCL. This combination was associated with greater disease control than R-CHOP/ $\mathrm{R}-\mathrm{CVP}$ with fewer patients requiring second-line therapy. However, response rates and PFS are not a surrogate for OS in the setting of low-grade lymphomas, and thus they represent an incomplete measure of clinical benefit. In both the BRIGHT and StiL-1 studies, a significant improvement in OS was not observed suggesting that the order in which BR and R-CHOP or R-CVP are administered could not be that important. Whether the use of BR will affect the natural history of these diseases is to be proven. The diverse toxicity profile, as the decision or not to proceed to maintenance treatment when indicated, may drive the physician choice together with patient preferences.

The era we are entering in, it will be characterized by the integration of targeted therapies with gene mutations sometimes driving the "single patient" choice of treatment, as whole exome sequencing profiling is becoming readily available, with acceptable costs if performed by experienced laboratories. Certainly, the availability of new targeted drugs to be integrated in future trials will improve our capability of understanding which regimen is actually more effective when given upfront, while increasing life expectancies of any of the included histologies. Translational research has the duty to improve our understanding and definition of "high biological risk", paving the way to precision medicine trials or single patient adapted therapies.

\section{Acknowledgments}

Funding: None.

\section{Footnote}

Conflicts of Interest: All authors have completed the ICMJE uniform disclosure form (available at http://dx.doi. org/10.21037/cco.2019.10.03). The authors have no conflicts of interest to declare.

Ethical Statement: The authors are accountable for all aspects of the work in ensuring that questions related to the accuracy or integrity of any part of the work are appropriately investigated and resolved.

Open Access Statement: This is an Open Access article distributed in accordance with the Creative Commons Attribution-NonCommercial-NoDerivs 4.0 International License (CC BY-NC-ND 4.0), which permits the noncommercial replication and distribution of the article with the strict proviso that no changes or edits are made and the original work is properly cited (including links to both the formal publication through the relevant DOI and the license). See: https://creativecommons.org/licenses/by-nc-nd/4.0/.

\section{References}

1. Flinn IW, van der Jagt R, Kahl BS, et al. Randomized trial of bendamustine-rituximab or R-CHOP/R-CVP in firstline treatment of indolent NHL or MCL: the BRIGHT study. Blood 2014;123:2944-52.

2. Rummel MJ, Niederle N, Maschmeyer G, et al. Bendamustine plus rituximab versus $\mathrm{CHOP}$ plus rituximab as first-line treatment for patients with indolent and mantle-cell lymphomas: an open-label, multicentre, randomised, phase 3 non-inferiority trial. Lancet 2013;381:1203-10.

3. Flinn IW, van der Jagt R, Kahl B, et al. First-Line Treatment of Patients With Indolent Non-Hodgkin Lymphoma or Mantle-Cell Lymphoma With Bendamustine Plus Rituximab Versus R-CHOP or R-CVP: Results of the BRIGHT 5-Year Follow-Up Study. J Clin Oncol 2019;37:984-91.

4. Salles G, Seymour JF, Offner F, et al. Rituximab maintenance for 2 years in patients with high tumour burden follicular lymphoma responding to rituximab plus chemotherapy (PRIMA): a phase 3, randomised controlled 
trial. Lancet 2011;377:42-51.

5. Rummel MJ, Viardot A, Greil R, et al. Bendamustine Plus Rituximab Followed By Rituximab Maintenance for Patients with Untreated Advanced Follicular Lymphomas. Results from the StiL NHL 7-2008 Trial (MAINTAIN trial) (ClinicalTrials.gov Identifier: NCT00877214). Blood 2014;124:3052.

6. Marcus R, Davies A, Ando K, et al. Obinutuzumab for the First-Line Treatment of Follicular Lymphoma. N Engl J Med 2017;377:1331-44.

7. Kluin-Nelemans HC, Hoster E, Hermine O, et al. Treatment of older patients with mantle-cell lymphoma. N Engl J Med 2012;367:520-31.

8. Rummel MJ, Knauf W, Goerner M, et al. Two years rituximab maintenance vs. observation after first-line treatment with bendamustine plus rituximab (B-R) in patients with mantle cell lymphoma: First results of a prospective, randomized, multicenter phase II study (a subgroup study of the StiL NHL7-2008 MAINTAIN

Cite this article as: Visco C, Quaglia FM, Bovo C, Tisi MC, Krampera M. Bendamustine plus rituximab: is it a BRIGHT idea? Chin Clin Oncol 2020;9(2):22. doi: 10.21037/ cco.2019.10.03 trial). J Clin Oncol 2016;34:7503.

9. Albertsson-Lindblad A, Kolstad A, Laurell A, et al. Lenalidomide-bendamustine-rituximab in patients older than 65 years with untreated mantle cell lymphoma. Blood 2016;128:1814-20.

10. Visco C, Chiappella A, Nassi L, et al. Rituximab, bendamustine, and low-dose cytarabine as induction therapy in elderly patients with mantle cell lymphoma: a multicentre, phase 2 trial from Fondazione Italiana Linfomi. Lancet Haematol 2017;4:e15-23.

11. Salar A, Domingo-Domenech E, Panizo C, et al. Long-term results of a phase 2 study of rituximab and bendamustine for mucosa-associated lymphoid tissue lymphoma. Blood 2017;130:1772-4.

12. Iannitto E, Bellei M, Amorim S, et al. Efficacy of bendamustine and rituximab in splenic marginal zone lymphoma: results from the phase II BRISMA/IELSG36 study. Br J Haematol 2018;183:755-65. 